\title{
Premier CFC bientôt délivré
}

\section{Marie-Paule Fauchère ${ }^{a}$; Anne-Claude Perrette ${ }^{b}$}

${ }^{a}$ Marie-Paule Fauchère, présidente de I'Association romande des assistantes médicales (ARAM); ${ }^{b}$ Anne-Claude Perrette, cheffe experte Validation des acquis (VAE)

\section{Introduction}

Fin 2010, un groupe de travail romand «VAE pour assistant-e médical-e» a été fondé sur mandat de la FMH. Il a élaboré le Profil de qualification pour assistant-e médical-e et les conditions de réussite selon les normes OFFT (aujourd'hui Secrétariat d'Etat à la formation, à la recherche et à l'innovation [SEFRI]). Il a aussi créé, sur la base de compétences opérationnelles définies, l'architecture modulaire pour les compléments de formation des personnes en VAE.

\section{Historique du projet}

- Avant 2006: pratique à Genève et groupe de travail en Valais

- Mai 2007: entrée en vigueur du «Guide National VAE»

- Dès 2007: reconnaissance des deux dispositifs cantonaux Genève et Valais

- Dès 2010: groupe intercantonal GE-VD-VS constitué des partenaires institutionnels

- Dès 2011:

- groupe de travail «Profil de qualification»

- groupe de travail interrégional pour la création de l'offre de formation modulaire

- Décembre 2014: approbation du Profil de qualification et des conditions de réussite par le SEFRI

Les démarches suivantes ont été de:

- définir la collaboration et la coordination au niveau romand

- identifier les besoins au niveau cantonal (questionnaire auprès des cabinets médicaux)

- mettre en place le dispositif:

- nomination et formation des experts et de la cheffe experte VAE

- création des outils VAE (dossier, rapport d'expert, entretien)

- information des candidats en attente

- information des employeurs

- formation complémentaire (module radiologie)

Pour la Romandie (excepté Genève), l'organisation a été confiée au canton du Valais. L'inscription s'effectue par le portail d'entrée du canton de domicile du candidat. L'Association romande des assistantes médicales
(ARAM) collabore étroitement avec l'Office d'orientation scolaire et professionnelle du Valais romand, ValForm.

En 2014, création d'une commission professionnelle de validation. Elle est constituée d'un:

- représentant de l'école AMAD, école professionnelle pour AM à Sion

- représentant des employeurs, Société médicale du Valais

- représentant des employés, ARAM

- représentant des experts, cheffe experte ARAM

- représentant de la formation continue des adultes

- représentant de l'orientation professionnelle

- conseiller en bilan et référent de la profession

Des séances d'information ont été organisées. 6 personnes ont débuté la procédure en Valais, dont une Fribourgeoise et une Vaudoise. Elles disposent des profils suivants:

- CFC d'ASSC

- diplôme de nurse

- diplôme d'études commerciales + diplôme d'assistante médicale (école privée)

- CFC d'assistante dentaire SSO

- diplôme de secrétaire médicale (Culture et Formation, Fribourg)

Ces personnes ont été accompagnées par Val-Form pour l'élaboration du profil de qualification.

L'ARAM a proposé Mme Anne-Claude Perrette au poste de cheffe experte. Cette dernière a eu entre autres pour tâche de constituer un collège d'experts qui est composé de:

- 7 assistantes médicales, dont 5 polyvalentes

- 6 enseignants

Les experts ont suivi une formation de 2 jours en présentiel et bénéficieront d'un accompagnement post-formation.

Objectifs des cours:

- connaître l'histoire et la philosophie de la VAE

- connaître le principe de fonctionnement général de la procédure Val-Form

- prendre conscience de la problématique de l'adulte en formation 
- connaître les modalités d'évaluation et le rôle de l'évaluateur

- analyser un dossier de preuves

- mener un entretien de vérification

- inférer des compétences à partir de l'expérience

- rédiger un rapport d'évaluation

\section{Situation actuelle}

Correspondance:

Marie-Paule Fauchère

Rte de Saxonne 20

CH-1966 Ayent

presidente[at]aram-vd.ch

Anne-Claude Perrette

Ch. de Charbonaille 3

CH-1868 Les Neyres

acperrette[at]bluewin.ch
A ce jour, 4 personnes ont terminé le dossier de validation et le travail d'expertise va débuter:

- analyse et examen du dossier

- rédaction du rapport d'évaluation

- expertise en collège d'experts (l'expert principal peut solliciter l'avis d'un expert spécialiste)

- entretien de vérification d'environ $1 \frac{1}{2}$ heure. Si celui-ci ne s'avère pas suffisant, des méthodes de véri- fication supplémentaires peuvent être appliquées (mises en situation, tests, observation in situ, etc.)

- rapport final d'évaluation

- validation des modules. Pour les modules non acquis, des compléments de formation pour les domaines de qualification non acquis sont prévus.

Pour rappel: Pour des raisons juridiques, la radiologie ne peut pas faire l'objet d'une validation (formation et examen obligatoires).

Pour de plus amples informations concernant la validation des acquis (VAE), veuillez consulter www.am-suisse.ch $\rightarrow$ Validation des acquis et www.aram-vd.ch $\rightarrow$ Formation $\rightarrow$ Validation des acquis. 